\title{
ESTIMACIÓN DEL POTENCIAL ENERGÉTICO DE LOS PINARES DE LA PROVINCIA DE TERUEL MEDIANTE TELEDETECCIÓN
}

\author{
Alberto García-Martín, Juan de la Riva, Fernando Pérez-Cabello \\ y Raquel Montorio \\ Dpto. de Geografía y Ordenación del Territorio \\ Facultad de Filosofía y Letras, Universidad de Zaragoza \\ algarcia@unizar.es, delariva@unizar.es, fcabello@unizar.es,montorio@unizar.es
}

\begin{abstract}
Resumen: El aprovechamiento energético de la biomasa residual forestal presenta múltiples beneficios medioambientales y socioeconómicos; sin embargo, la falta de una metodología para estimar la cantidad presente a escala regional es una de las razones que impiden la mayor utilización de este recurso renovable en España. El presente trabajo presenta una metodología para evaluar la biomasa residual de los pinares de la provincia de Teruel (España) relacionando datos de esta fracción de biomasa calculados sobre parcelas de inventario forestal de 1994 con una imagen Landsat coetánea a las labores de campo del inventario. Para evitar la influencia que la heterogeneidad de los medios forestales mediterráneos tiene en el ajuste de modelos de regresión, se ensayan tres métodos distintos de extracción de la información espectral. Una vez validadas las ecuaciones obtenidas con estos tres métodos, la mejor es aplicada sobre una imagen Landsat más reciente. Se obtiene así la cantidad potencial de este recurso (5.449.252 tons) y cartografía precisa sobre su distribución en el territorio turolense.
\end{abstract}

Palabras clave: biomasa residual forestal, imágenes Landsat, modelos de regresión, Teruel (España).

\begin{abstract}
The use of forest residual biomass for energy production shows many environmental and socio-economic benefits. However, the lack of a methodology to assess the regional-scale quantity of residual biomass is one of the main factors that hamper the increase in the use of this renewable resource in Spain. The present work shows a method-
\end{abstract}

Recibido: 9-11-10. Aceptado: 2-2-12. 
ology to evaluate the forest residual biomass present in the pine forest of Teruel province (Spain) relating data of this biomass fraction calculated in the 1994 forest inventory with a Landsat image selected on the basis of its temporal coincidence with the inventory fieldwork. In order to avoid the influence that the heterogeneity of Mediterranean forest leads in the performance of predictive models, three different methods to extract the radiometric data are tested. The regression models derived were validated and the best equation was applied to a recent Landsat image. As a result, the potential quantity of this resource (5.449.252 tons) and an accurate distribution cartography of Teruel province were obtained.

Keywords: forest residual biomass, Landsat images, regression models, Teruel (Spain).

\section{Introducción}

El concepto de biomasa residual forestal (BRF) engloba al conjunto formado por hojas-acículas, ramas y raberón (parte superior del tallo con diámetro entre $5-10 \mathrm{~cm}$ ) generado tanto en tratamientos selvícolas como en aprovechamientos madereros parciales o finales que no son extraídos habitualmente por no ser convertibles en subproductos, pero que pueden ser utilizados como combustible orgánico en aplicaciones eléctricas, térmicas y de cogeneración (Esteban et al., 2004, Velázquez, 2006). El aprovechamiento energético de estos residuos, que de forma general son amontonados en el monte hasta su descomposición o quemados in situ de forma controlada (Velázquez, 2006), tiene un impacto ambiental positivo, dado que su retirada hace disminuir el riesgo de incendio forestal y la severidad de éstos, permite mejorar el estado de las masas forestales y su combustión presenta un balance neutro en el ciclo del $\mathrm{CO}_{2}$. Por otro lado, la valorización de la BRF presenta beneficios económicos y sociales a escala local de forma directa e indirecta, ya que la aparición de empresas para la generación, recolección, transporte y aprovechamiento, además de aumentar la oferta de empleo, puede suponer también un importante impulso a la mejora de infraestructuras y la diversificación de la actividad económica del espacio rural. Además, a escala nacional, contribuye a reducir la dependencia energética del petróleo (Richardson et al., 2002). Sin embargo, el incumplimiento de los objetivos marcados en producción de energía renovable a mediados de la década pasada llevó a la revisión del vigente Plan de Fomento de las Energías Renovables en España. En él se destacó como una de las principales barreras que impedía el crecimiento en el uso de los residuos forestales el desconocimiento de la capacidad real de producción 
de BRF de una masa forestal (IDAE, 2005). La determinación de una metodología que permita superar esta barrera es una cuestión esencial, ya que el primer paso para el crecimiento de esta fuente de energía debe ser el conocimiento de la cantidad de recursos disponibles para ser usados (Esteban et al., 2004; Velázquez, 2006).

Numerosos trabajos han mostrado el potencial de las imágenes de satélite para las tareas de inventario forestal, permitiendo el carácter continuo, completo y recurrente de éstas superar las limitaciones de otros métodos de inventario basados exclusivamente en el trabajo de campo y la interpolación de datos puntuales y de técnicas SIG (Salvador y Pons, 1998, Franklin, 2001). Así, la teledetección ha contribuido a incrementar la velocidad, la eficiencia de coste y la precisión de los inventarios (McRoberts y Tomppo, 2007), convirtiéndose en una fuente primaria para la estimación de biomasa (Lu, 2006). Sin embargo, ninguna de las aproximaciones desarrolladas para estimar biomasa ha logrado presentar una técnica consistente y enteramente reproducible a escala regional o continental (Muukkonen y Heiskanen, 2005), encontrándose los principales problemas en bosques localizados en zonas de topografía complicada caracterizados por la presencia de múltiples especies y diferentes rangos de edad y con una estructura compleja (Lu, 2006); éstas son características inherentes a los bosques mediterráneos (Shoshany, 2000), medios en los que las experiencias desarrolladas son menores. En este contexto, el objetivo de este trabajo es conocer la cantidad potencial de BRF mediante el uso de imágenes de satélite e información forestal preexistente en los bosques de pináceas de la provincia de Teruel.

\section{2. Área de estudio}

La elección de la provincia de Teruel (España) como área de estudio se decidió en el contexto del proyecto LIGNOSTRUM (CICyT AGL2002-03917-AGR-FOR), en el cual se insertó el presente trabajo. Los motivos para seleccionar esta provincia fueron dos: la presencia de una importante superficie forestal ( $405.502 \mathrm{ha}, 27 \%$ del territorio) y la existencia de espacios rurales económicamente desfavorecidos. Tres son las características de esta provincia con una alta incidencia para cumplir el objetivo: (i) su elevada extensión $\left(14.804 \mathrm{~km}^{2}\right.$ ), (ii) la existencia de una topografía compleja y (iii) el carácter mediterráneo de sus bosques. Estas particularidades determinan el tipo de imagen a utilizar, los tratamientos a aplicar y el método de extracción de la información espectral. Entre los tipos de bosque existentes, se seleccionaron los pinares de Pinus sylvestris, P. halepensis, P. nigra y P. pinaster, dado que éstos representan el $71 \%$ de la superficie forestal provincial y son los que tienen el mayor potencial para la generación de residuos. 


\section{Materiales y métodos}

\subsection{Los datos de biomasa residual}

La información de partida para calcular los datos de BRF fueron las parcelas del Segundo Inventario Forestal Nacional (IFN-2) de Teruel. Estas parcelas, de forma circular con un radio de entre $5-25 \mathrm{~m}$, se encuentran localizadas en los cruces de la cuadrícula UTM 1:50.000, siendo su ubicación exacta en el campo hecha mediante cartografía topográfica y ortofotografías 1:30.000. Los trabajos levantamiento y medición de estas parcelas se realizaron entre marzo y agosto de 1994. Sobre estas parcelas se calculó la BRF mediante regresiones específicas de cada especie desarrolladas en el proyecto LIGNOSTRUM a partir de muestreo destructivo de campo. En concreto, se muestrearon 186 pies (30 de P. sylvestris, 59 de P. halepensis, 57 de P. nigra y 40 de $P$. pinaster). Para cada árbol, se obtuvo el peso húmedo de la BRF, el diámetro a la altura del pecho (dbh) y la altura y se recogieron muestras de hojas y ramas para calcular el peso seco. Todas las regresiones ajustadas presentaron un $\mathrm{R}^{2}$ superior a 0,90 (Alonso et al. 2005). Para evitar inconsistencias en los datos de cada parcela y en el ajuste de los modelos de estimación debido a la mezcla de signaturas espectrales, las regresiones sólo se aplicaron a parcelas monoespecíficas en las que los pies incluidos estaban dentro del rango muestreado, obteniéndose de esta forma información expresada en tons/ha en un total de 482 parcelas.

\subsection{Las imágenes de satélite}

De entre los distintos tipos disponibles se seleccionaron imágenes Landsat, las más utilizadas en aplicaciones de teledetección en tareas de inventario forestal y de estimación de biomasa a escalas regional y local (Lu, 2006). Junto a esto, otros motivos fueron la obtención mediante una sola escena de la mayor parte del territorio del área de estudio, evitando de esta forma errores debidos a inconsistencias en procesos de corrección geométrica y radiométrica de varias escenas, y el carácter periódico de su cobertura y su fácil adquisición, lo que permite que la metodología creada sea replicable en otros territorios. En concreto, se utilizaron dos imágenes Landsat TM de la misma orbita de observación terrestre y fila (track 199, frame 32), pero registradas en momentos diferentes: el 29 de junio de 1994 y el 5 de julio de 2008. La primera de ellas, coetánea a los trabajos de campo del IFN-2 en Teruel, fue utilizada para el ajuste de los modelos de estimación de la BRF, mientras que la segunda fue reservada para aplicar el modelo mejor ajustado y obtener así la cartografía actual del recurso. La utilización de imágenes de verano -las más habituales en trabajos forestales con teledetección- minimiza el efecto de las sombras en la información registrada; en todo caso, estudios previos han puesto en evidencia la buena relación existente entre la variable estudiada y los datos Landsat independientemente de la fecha de adquisición 
de la imagen dentro del periodo estival (García-Martín et al., 2008). Al objeto de obtener variables espectrales consistentes y susceptibles de ser relacionadas con los datos de campo de BRF y para aplicar los modelos de estimación ajustados con la imagen de 1994 sobre la de 2008, se aplicaron tres pretratamientos sobre ambas: (i) corrección geométrica, en la que aplicó un remuestreo a $25 \mathrm{~m}$ de resolución espacial con un error inferior a un píxel; (ii) corrección radiométrica, mediente el método de Minnaert (Colby, 1991); y (iii) aplicación de transformaciones, para la obtención de nuevas bandas que realzaran algunos rasgos de interés de las imágenes: análisis de componentes principales, Tasseled-Cap e índices de vegetación.

\subsection{Métodos de extracción de la información espectral}

Como se ha indicado, la heterogeneidad de los bosques mediterráneos es uno de los principales factores que dificulta la estimación de parámetros forestales en estos medios. Esto es debido a que esta heterogeneidad espacial se traduce en una elevada variabilidad espectral de las áreas ocupadas por estos bosques. Otros factores son el pequeño tamaño de las parcelas, la comisión de imprecisiones en su localización y el escaso número de parcelas usados (Salvador y Pons, 1998). Así, en el caso concreto de las parcelas utilizadas, dos de ellas con una misma cantidad de BRF pueden presentar valores medios de reflectividad distintos debido a la presencia de otro tipo de cubierta o a una alta variabilidad interna. Para superar estos problemas, se ensayaron 3 métodos para extraer la información espectral: (i) ventanas fijas, (ii) análisis visual, y (iii) segmentación espectral.

La utilización de ventanas fijas de tamaño superior a un píxel es la forma de proceder más recurrente en los trabajos que utilizan imágenes ópticas de resolución media para la estimación de parámetros forestales. Para este trabajo, se utilizaron ventanas de $3 \times 3$ píxeles situadas sobre el centro de las 482 parcelas con datos, extrayéndose información de la media y de la desviación estándar en cada variable espectral. Para obtener una medida de la variabilidad espectral de cada parcela y evaluar la influencia de ésta en el ajuste de los modelos de estimación, se calculó el coeficiente de variación de Pearson (CV) con la información obtenida de las 6 bandas reflectivas. Los valores obtenidos de CV fueron utilizados para crear 10 grupos de parcelas, de tal manera que cada grupo fuera incluyendo un número menor, pero con un entorno radiométrico más homogéneo. Para ello, en primer lugar, se calculó para cada banda reflectiva los umbrales de CV que permitían la separación de las parcelas en 10 percentiles. Con posterioridad, esos umbrales sirvieron para la delimitación de los grupos de tal manera que el primero incluye todas las parcelas, el segundo aquellas con $\mathrm{CV}$ inferiores a los valores del percentil 9 en todas las bandas reflectivas, el tercero aquellas cuyos CV estaban por debajo de los valores de CV del percentil 8 y así sucesivamente hasta delimitar 10 grupos. 
La aplicación de los otros dos métodos de extracción trata de soslayar algunos de los problemas del método de ventanas fijas que ocasiona imprecisiones en los resultados, ya que este método no elimina completamente los errores relacionados con la localización de las parcelas en el campo, y/o con la georreferenciación de la imagen, que pueden intersectar otras unidades de características espectrales y forestales diferentes a la de la parcela y la información de media hace que se pierdan detalles específicos (Mäkkelä y Pekkarinen, 2001; Muukkonen y Heiskanen, 2005). Junto a esto, otro inconveniente es que la utilización de información por parcelas puede no ser representativa como nivel de análisis para toda la imagen de satélite, ya que no representa unidades homogéneas desde el punto de vista espectral (Mäkkelä y Pekkarinen, 2001). Una solución a estos problemas es utilizar como método de extracción unidades de mayor homogeneidad y tamaño que las parcelas para las cuales se tienen datos. Estas unidades deben ser homogéneas desde el punto de vista de sus características forestales (Mäkelä y Pekkarinen, 2004; Muukkonen y Heiskanen, 2005) o espectrales (Mäkelä y Pekkarinen, 2001). Teniendo en cuenta esto, el segundo método de extracción se basó en la utilización de áreas homogéneas forestales de igual composición y estructura que las parcelas del inventario, siendo éstas generadas a partir de la interpretación de fotografía aérea de alta resolución espacial $(1 \mathrm{~m})$ perteneciente al vuelo del SIG Oleícola en Aragón (1998). A cada nueva área homogénea se le asignó un indicador del grado de similitud observado en la fotografía aérea entre la parcela IFN-2 analizada y su nueva delineación: 0, nula similitud; 1, similitud baja; 2, similitud media; 3, similitud alta. Este indicador se utiliza para crear tres grupos de áreas homogéneas sobre los que se efectúa el análisis estadístico: grupo 1 (compuesto por las áreas de similitud baja, media y alta), grupo 2 (áreas de similitud media y alta) y grupo 3 (sólo áreas de similitud alta). La información de media de cada una de las bandas fue obtenida utilizando solamente los píxeles que quedaban completamente dentro de los nuevos límites. Por su parte, las áreas espectrales homogéneas utilizadas en el tercer método de extracción fueron creadas a partir del algoritmo de segmentación $R G B$ clustering implementado en Erdas Imagine. Los parámetros esenciales que controlan los resultados de este método son la composición de color sobre la que se aplica el proceso, el escalado de cada uno de los histogramas de esas bandas y el número de secciones a considerar en cada una de ellas. Se ensayaron 4 segmentaciones diferentes para modelizar la heterogeneidad de los bosques analizados $\left(\mathrm{S}_{1}, \mathrm{~S}_{2}, \mathrm{~S}_{3}, \mathrm{~S}_{4}\right)$, modificando los parámetros de escalado del histograma y del número de secciones sobre una composición RGB TM7-TM4-TM3, siendo ésta la seleccionada en función de criterios visuales y estadísticos a la hora de estimar biomasa residual (García Martín, 2009). Finalmente, para evitar introducir errores relacionados con la localización de parcelas y evitar el uso de píxeles no representativos, sólo se extrajo la información espectral (media) de los píxeles de la ventana de 3x3 que pertenecían a la misma área espectral homogénea que el píxel central de la parcela del IFN-2. 


\subsection{Ajuste de los modelos, validación y aplicación para el inventario}

Para explorar la posibilidad de establecer modelos de estimación operativos y representativos para todo el área de estudio con los distintos grupos de parcelas creados se utilizó el coeficiente de correlación de Pearson. Una vez seleccionados estos grupos, la muestra de parcelas se dividió en dos, siendo el 80\% utilizadas para el ajuste del modelo y el $20 \%$ para completar la valoración de la exactitud de la predicción. Además, para valorar la robustez del modelo, la división de la muestra se realizó de forma aleatoria en cinco ocasiones $\left(\mathrm{N}_{1}, \mathrm{~N}_{2}, \mathrm{~N}_{3}, \mathrm{~N}_{4}\right.$ y $\left.\mathrm{N}_{5}\right)$, calculándose el respectivo modelo y la validación en cada una de ellas (Tablas 2, 4 y 6). El método de ajuste de los modelos fue el de regresión lineal múltiple por "pasos hacia delante", incluyéndose solamente las variables con significación estadística $(p<0,05)$ y verificándose el cumplimiento de los supuestos básicos de este tipo de regresión tanto a nivel de variable como de modelo. Para valorar la exactitud de la predicción se utilizaron el coeficiente de determinación ajustado $\left(\mathrm{R}_{\mathrm{a}}{ }_{\mathrm{a}}\right)$ de los modelos ajustados y el

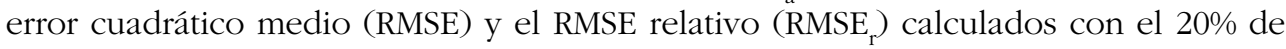
las parcelas no utilizadas en el ajuste de cada ecuación. Finalmente, el mejor de los cinco modelos ajustados en cada uno de los tres métodos fue aplicado sobre la imagen de junio de 1994 para obtener cartografía de estimación. Las tres cartografías obtenidas son validadas mediante el uso de las parcelas no incluidas en los grupos considerados, calculándose el RMSE y el RMSE $\mathrm{r}_{\mathrm{r}}$. A partir de los resultados obtenidos a nivel de modelo y de la validación de la cartografía obtenida en su aplicación, la ecuación de regresión más adecuada es seleccionada para ser aplicada sobre la imagen de julio de 2008, obteniéndose de esta forma información actual sobre la cantidad potencial de este recurso energético y sobre su distribución espacial a escala provincial.

\section{Resultados}

\subsection{Modelos con ventanas fijas}

Como se esperaba, la correlación entre la BRF y las variables espectrales consideradas fue más alta a medida que los 10 grupos delimitados usando los CV contenían un menor número parcelas y una variabilidad espectral menor (Tabla 1). Todos los grupos presentaron correlaciones significativas con la gran mayoría de variables $(p<0,05)$, con la única excepción del grupo del percentil 1 debido a que sólo contenía 3 parcelas. En todos los grupos, los mejores coeficientes de correlación se obtuvieron con las variables relacionadas - directa o inversamente- con la humedad de la vegetación (TM5, TM7, TC3, MSI y MID57), alcanzando valores 


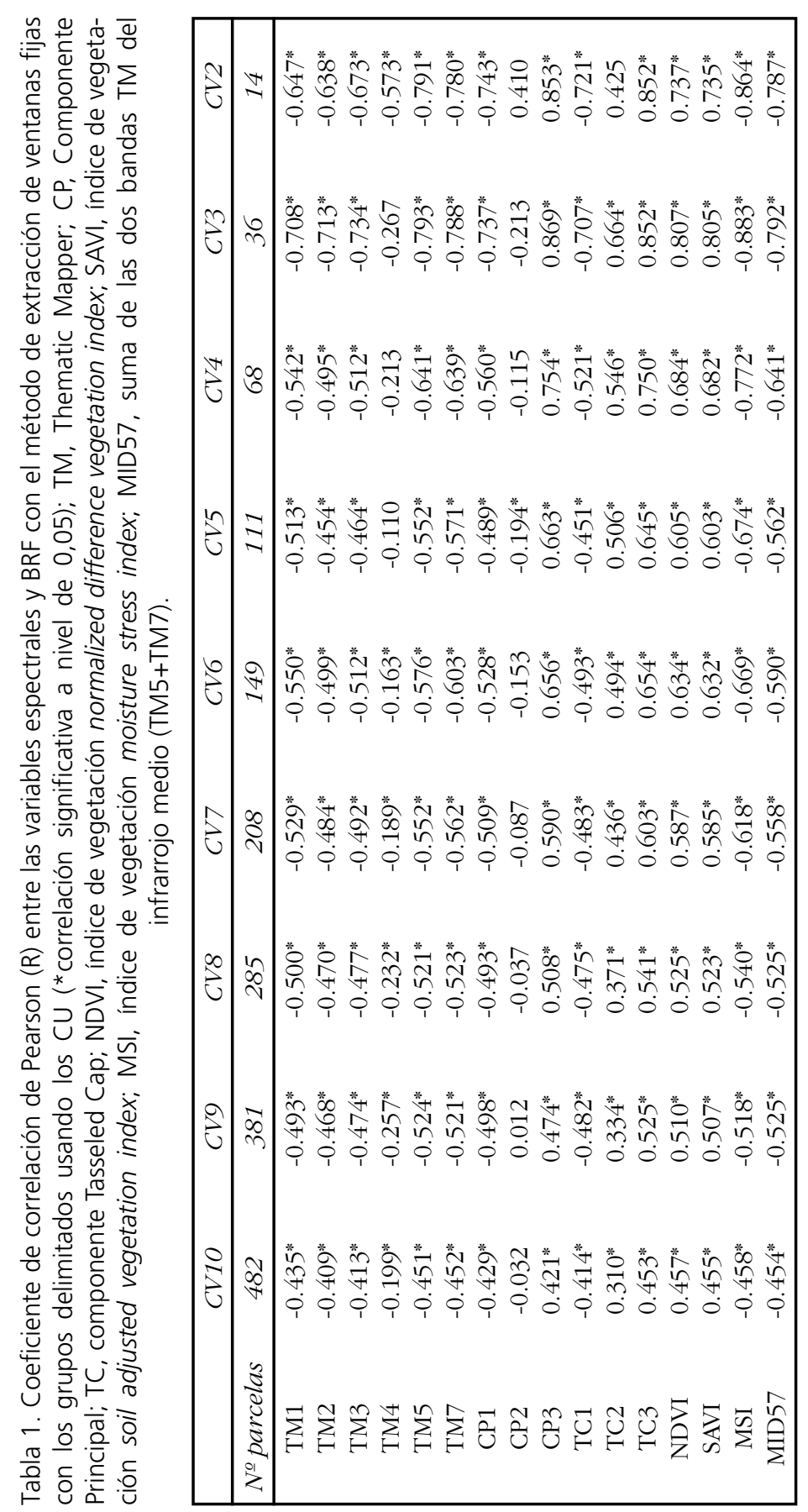


similares el índice de vegetación NDVI. Estos coeficientes pasan de valores comprendidos entre 0,4 y 0,5 en el grupo 10, que contiene todas las parcelas, hasta valores $>0,75$ a partir del grupo del percentil 3. De los resultados de este análisis se desprende que la heterogeneidad espectral condiciona la posibilidad de establecer modelos precisos, dado que si se utilizan los grupos que contienen más parcelas, los modelos presentarán una capacidad predictiva baja, mientras que, si se emplean los que menor número de parcelas tienen, existe la posibilidad de obtener modelos con una alta capacidad predictiva pero con un alto riesgo de estar sobre-ajustados a la muestra y, por tanto, de no ser representativos de todos los bosques de la provincia de Teruel. Teniendo en cuenta esto, se seleccionó el grupo de parcelas correspondiente al percentil 4, ya que dentro de los grupos caracterizados por una alta homogeneidad de las parcelas contenidas que permite ajustar modelos con un alto $\mathrm{R}^{2}$, éste es el que mayor número de parcelas presentaba (68). La visualización de gráficos de dispersión entre la BRF y cada una de estas variables mostró el carácter no lineal de muchas de estas relaciones. En consecuencia, se aplicó tanto a la variable independiente como a las dependientes la transformación estandarizada más adecuada para garantizar el supuesto de linealidad que exige la regresión lineal (aplicación de logaritmos -ln-, raíz cuadrada -rz-, cuadrado, -cd- e inversa -inv-) (Hair et al., 1999). La Tabla 2 muestra los modelos de regresión lineal ajustados con cada una de las divisiones aleatorias de la muestra. Todos ellos incluyen solamente una variable, estando siempre ésta relacionada con el contenido de agua de la vegetación. La no incorporación de más variables en ninguno de los modelos se debió a la alta auto-correlación existente entre ellas, lo que hace que no se cumpla el supuesto de no-colinealidad de la regresión múltiple. Aun así, los $\mathrm{R}^{2}$ alcanzados, superiores a 0,7, indican su utilidad para estimar la BRF del área de estudio. Los resultados obtenidos con la muestra reservada del 20\% señalaron al ajustado con la variable MID57 (suma de las bandas del infrarrojo medio de onda corta, TM5+TM7) como el más adecuado, ya que presenta un RMSE $_{r}$ de sólo el 26,67\%, significativamente inferior al resto, por lo que fue el seleccionado para obtener cartografía.

Tabla 2. Modelos de regresión con el método de extracción de ventanas fijas con el grupo de parcelas correspondiente al percentil 4 (variable dependiente: raíz de BRF).

\begin{tabular}{|ccccccc|}
\hline $\begin{array}{c}\text { Modelo- } \\
\text { Muestra }\end{array}$ & Variable & $R^{2} a$ & $\beta_{0}$ & $\beta_{1}$ & $\begin{array}{c}\text { RMSE } \\
\text { (tons/ha })\end{array}$ & $\begin{array}{c}\text { RMSEr } \\
(\%)\end{array}$ \\
\hline $\mathrm{N}_{1}$ & ln_MID57 & 0,711 & 18,879 & $-4,663$ & 4,843 & 26,67 \\
$\mathrm{~N}_{2}$ & $\ln \_$TM5 & 0,713 & 17,933 & $-5,073$ & 4,591 & 43,38 \\
$\mathrm{~N}_{3}$ & ln_TM5 & 0,750 & 17,900 & $-5,053$ & 4,767 & 51,91 \\
$\mathrm{~N}_{4}$ & ln_TM7 & 0,735 & 12,603 & $-3,906$ & 6,144 & 42,92 \\
$\mathrm{~N}_{5}$ & ln_TM5 & 0,750 & 18,253 & $-5,204$ & 4,792 & 34,93 \\
\hline
\end{tabular}




\subsection{Modelos con áreas forestales homogéneas}

El grado de correlación entre la BRF y las variables aumentó conforme lo hacía la similitud de las nuevas áreas forestales creadas y lo observado dentro de las parcelas del IFN-2, siendo casi todas ellas significativas (Tabla 3). Independientemente de esto, las variables relacionadas con la humedad se volvieron a mostrar como las más correlacionadas. Así, los resultados obtenidos indicaron que solamente es posible ajustar modelos con la misma capacidad predictiva que los obtenidos con el método de extracción de las ventanas de 3x3 utilizando las áreas homogéneas visuales del grupo 3 (similitud alta), ya que los coeficientes de correlación alcanzados eran similares a los obtenidos con el grupo del percentil 4 utilizado con anterioridad $(\mathrm{R}>0,7)$. Sin embargo, dado que el número de parcelas incluidas en este grupo 3 es el doble que en grupo del percentil 4 (131 contra 68), se reduce la posibilidad de obtener modelos sobre-ajustados a la muestra (Hair et al., 1999). Solo uno de los 5 modelos ajustados logró incorporar más de una variable (Tabla 4) debido, como en el caso anterior, a la alta autocorrelación existente entre las variables espectrales. Esta circunstancia hace que el método de regresión por pasos seleccione en cada división la variable más relacionada con la BRF, siendo siempre ésta una relacionada con el contenido de humedad. Para derivar cartografía se seleccionó el modelo ajustado con la muestra $\mathrm{N}_{3}$, dado que presenta un buen compromiso entre la capacidad de predicción y el error obtenido y, además, permite la comparación directa con el método de extracción anterior, ya que utiliza la misma variable (MID57).

Tabla 3. Coeficiente de correlación de Pearson (R) entre las variables espectrales y BRF con el método de extracción de áreas forestales homogéneas ( ${ }^{*}$ correlación significativa a nivel de 0,05).

\begin{tabular}{|c|c|c|c|c|c|c|c|}
\hline & $\begin{array}{l}\text { Grupo } 1 \\
\text { (similitud } \\
1,2 y 3)\end{array}$ & $\begin{array}{c}\text { Grupo } 2 \\
\text { (similitud } \\
2 \text { y3) }\end{array}$ & $\begin{array}{c}\text { Grupo } 3 \\
\text { (similitud } \\
\text { 3) }\end{array}$ & & $\begin{array}{l}\text { Grupo } 1 \\
\text { (similitud } \\
1,2 y 3)\end{array}$ & $\begin{array}{l}\text { Grupo } 2 \\
\text { (similitud } \\
2 y 3 \text { ) }\end{array}$ & $\begin{array}{c}\text { Grupo } 3 \\
\text { (similitud } \\
3 \text { ) }\end{array}$ \\
\hline $\begin{array}{c}N^{o} \text { de } \\
\text { parcelas }\end{array}$ & 436 & 353 & 131 & $\begin{array}{c}N^{o} \text { de } \\
\text { parcelas }\end{array}$ & 436 & 353 & 131 \\
\hline TM1 & $-0,504^{*}$ & $-0,550^{*}$ & $-0,633^{*}$ & $\mathrm{CP} 3$ & $0,527^{*}$ & $0,577^{*}$ & $0,678^{*}$ \\
\hline TM2 & $-0,512^{*}$ & $-0,555^{*}$ & $-0,628^{*}$ & TC1 & $-0,531^{*}$ & $-0,577^{*}$ & $-0,648^{*}$ \\
\hline TM3 & $-0,523^{*}$ & $-0,567^{*}$ & $-0,638^{*}$ & $\mathrm{TC} 2$ & $0,387^{*}$ & $0,424^{*}$ & $0,537^{*}$ \\
\hline TM4 & $-0,266^{*}$ & $-0,285^{*}$ & $-0,330^{*}$ & TC3 & $0,581^{*}$ & $0,627^{*}$ & $0,708^{*}$ \\
\hline TM5 & $-0,575^{*}$ & $-0,622^{*}$ & $-0,693^{*}$ & NDVI & $0,529^{*}$ & $0,572^{*}$ & $0,652^{*}$ \\
\hline TM7 & $-0,569^{*}$ & $-0,613^{*}$ & $-0,688^{*}$ & SAVI & $0,527^{*}$ & $0,570^{*}$ & $0,651^{*}$ \\
\hline CP1 & $-0,548^{*}$ & $-0,594^{*}$ & $-0,665^{*}$ & MSI & $-0,562^{*}$ & $-0,611^{*}$ & $-0,708^{*}$ \\
\hline $\mathrm{CP} 2$ & $-0,069$ & $-0,076$ & $-0,174^{*}$ & MID57 & $-0,574^{*}$ & $-0,620^{*}$ & $-0,692^{*}$ \\
\hline
\end{tabular}


Tabla 4. Modelos de regresión con el método de extracción de áreas forestales homogéneas con el grupo de áreas correspondientes al grupo 3 (similitud alta) (variable dependiente: raíz de BRF).

\begin{tabular}{|cccccccc|}
\hline $\begin{array}{c}\text { Modelo- } \\
\text { Muestra }\end{array}$ & Variable/s & $R^{2} a$ & $\boldsymbol{\beta}_{0}$ & $\boldsymbol{\beta}_{1}$ & $\boldsymbol{\beta}_{2}$ & $\begin{array}{c}\text { RMSE } \\
\text { (tons/ha) }\end{array}$ & $\begin{array}{c}\text { RMSEr } \\
\text { (\%) }\end{array}$ \\
\hline $\mathrm{N}_{1}$ & ln_MID57 & 0,610 & 16,822 & $-3,960$ & - & 12,207 & 64,95 \\
$\mathrm{~N}_{2}$ & $\ln \_$TM5 & 0,562 & 16,625 & $-4,541$ & - & 7,497 & 41,78 \\
$\mathrm{~N}_{3}$ & ln_MID57 & 0,595 & 17,675 & $-4,191$ & - & 8,839 & 59,48 \\
$\mathrm{~N}_{4}$ & MSI, inv_TM4 & 0,579 & 6,649 & $-5,909$ & 45,999 & 8,079 & 54,32 \\
$\mathrm{~N}_{5}$ & ln_TM5 & 0,558 & 17,492 & $-4,838$ & - & 11,238 & 56,68 \\
\hline
\end{tabular}

\subsection{Modelos con áreas espectrales homogéneas}

El análisis de correlación de los datos obtenidos con este método sólo se llevó a cabo con los grupos del percentil 7 y 6 definidos a partir del uso de CV por dos motivos: (i) para descartar directamente del análisis las parcelas con mayor posibilidad de errores y (ii) al objeto de identificar un modelo de estimación que alcance $\mathrm{R}^{2}$ tan elevados como los obtenidos con el método de ventanas de $3 \times 3$ pero con un mayor número de parcelas, quedando así asegurada su representatividad para los bosques de toda la provincia (Tabla 5). Nuevamente, las variables relacionadas con la humedad de la vegetación alcanzaron los mayores coeficientes en todas las segmentaciones, incrementándose las correlaciones conforme lo hacía la homogeneidad de la muestra independientemente del tipo de segmentación considerado. Solamente la segmentación $\mathrm{S}_{4}$ consiguió superar en algunas variables los coeficientes obtenidos con una ventana de $3 \times 3$ sin restricciones. En concreto, el máximo valor se dio utilizando esta segmentación con las variables TM7 y MID57, siendo éste algo superior a 0,7. Cuatro de los modelos de regresión quedaron compuestos por una variable relacionada con la humedad, siendo también una de éstas la seleccionada en primer lugar en el único modelo múltiple ajustado (Tabla 6). Los $\mathrm{R}^{2}$ a obtenidos se situaron en el intervalo comprendido entre 0,53 y 0,59 y únicamente uno de los modelos presentó un $\mathrm{RMSE}_{\mathrm{r}}$ inferior al 40\%. Dada su escasa diferencia en el $\mathrm{R}^{2}$ con respecto al resto, este fue el seleccionado para derivar cartografía.

\subsection{Validación de las cartografías de BRF}

El grado de acierto de los modelos ajustados (RMSE y RMSE $\mathrm{r}_{\mathrm{r}}$ ha sido validado con parcelas que cumplían, en cada caso, los mismos criterios de homogeneidad que las empleadas en el ajuste. Sin embargo, dado que las ecuaciones seleccionadas han sido aplicadas a cada uno de los píxeles forestales de la imagen Landsat, el grado de 


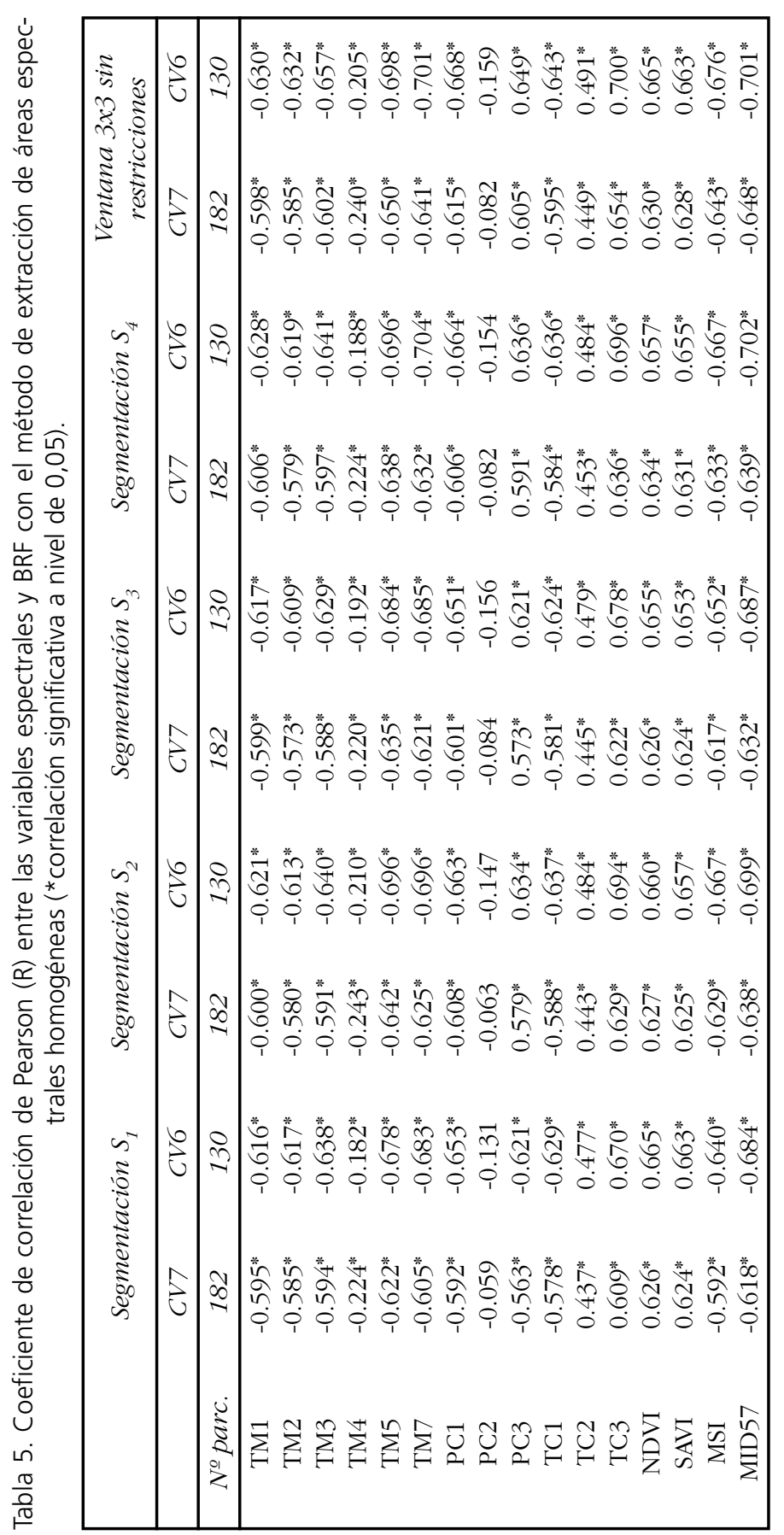


Tabla 6. Modelos de regresión con el método de extracción de áreas espectrales homogéneas con el grupo de parcelas correspondiente a la segmentación $S^{4}$ (variable dependiente: raíz de BRF).

\begin{tabular}{|cccccccc|}
\hline $\begin{array}{c}\text { Modelo- } \\
\text { Muestra }\end{array}$ & Variable/s & $R^{2} a$ & $\boldsymbol{\beta} 0$ & $\boldsymbol{\beta}_{1}$ & $\boldsymbol{\beta}_{2}$ & $\begin{array}{c}\text { RMSE } \\
\text { (tons/ha) }\end{array}$ & $\begin{array}{c}\text { RMSEr } \\
\text { (\%) }\end{array}$ \\
\hline $\mathrm{N}_{1}$ & TC3, In_TM1 & 0,547 & 7,746 & 0,303 & $-1,087$ & 9,064 & $44,95 \%$ \\
$\mathrm{~N}_{2}$ & ln_MID57 & 0,535 & 16,409 & $-3,888$ & - & 5,657 & 35,58 \\
$\mathrm{~N}_{3}$ & ln_TM7 & 0,560 & 11,395 & $-3,411$ & - & 8,840 & 50,07 \\
$\mathrm{~N}_{4}$ & ln_MID57 & 0,596 & 16,563 & $-3,928$ & - & 9,688 & 66,67 \\
$\mathrm{~N}_{5}$ & ln_MID57 & 0,533 & 16,217 & $-3,846$ & - & 8,231 & 51,01 \\
\hline
\end{tabular}

acierto en éstas tienen que ser evaluado también a esa escala. Para ello, se empleó el resto de las parcelas no utilizadas en cada ajuste, eliminando solamente aquellas que pudieran no ser óptimas para este propósito debido a errores de localización o por estar ubicadas en zonas muy heterogéneas. En concreto, se emplearon las parcelas del percentil 8, ya que éste incluye un número alto para garantizar que los resultados de validación no están sesgados por utilizar sólo las ideales.

Los resultados muestran las escasas diferencias existentes entre las tres cartografías, ya que la obtenida a partir de ventanas fijas de 3x3 arrojó un RMSE $_{\mathrm{r}}$ de $64,26 \%$, mientras que para las áreas forestales y espectrales homogéneas se situaron, respectivamente, en el 66,71\% y $65,06 \%$. Estos resultados a escala de píxel son considerados como tolerables para el área de estudio considerando experiencias previas llevadas a cabo empleando metodologías similares en ambientes boreales, menos afectados por la heterogeneidad que los bosques mediterráneos. Así, por ejemplo, los trabajos de Tokola (1996), Mäkkelä y Pekkarien (2001) y Katila y Tomppo (2001) obtuvieron -para estimaciones de volúmenes de madera o volúmenes totales- RMSE $_{\mathrm{r}}$ situados entre $65 \%$ y $100 \%$.

\subsection{Inventario de los residuos potencialmente disponibles}

Para inventariar la BRF se aplicó sobre el neocanal MID57 derivado de la imagen de julio de 2008 el modelo seleccionado en el método de extracción de ventanas fijas de $3 \times 3$. Esto es debido a que, por un lado, esta ecuación es, de las 3 utilizadas, la que presenta una mayor capacidad de predicción $\left(\mathrm{R}^{2}\right.$ de 0,711 frente a 0,595 del modelo seleccionado en el método de áreas homogéneas forestales y 0,535 en del método de áreas homogéneas espectrales) y, por otro, es el que permite, aunque ligeramente, obtener cartografía con el menor error de estimación. Este último hecho pone de manifiesto que el limitado conjunto de parcelas utilizado en el método de ventanas fijas de 3x3 es tan representativo para modelar la BRF como los utilizados en los otros dos métodos, a pesar de que éstos estaban compuestos por el doble de casos. 
La Figura 1 muestra la cartografía obtenida. Ésta, además de servir para calcular la cantidad total de este recurso a escala provincial, que asciende a 5.449.252 tons, permite conocer su distribución de forma precisa gracias a que está espacializada con una resolución de $25 \times 25 \mathrm{~m}$. Esto la convierte en muy adecuada para determinar zonas de aprovechamiento óptimo teniendo en cuenta otras variables espaciales que condicionan la viabilidad de su explotación como, por ejemplo, la pendiente, la distancia a pistas y la extensión del área forestal (Pascual et al., 2007).

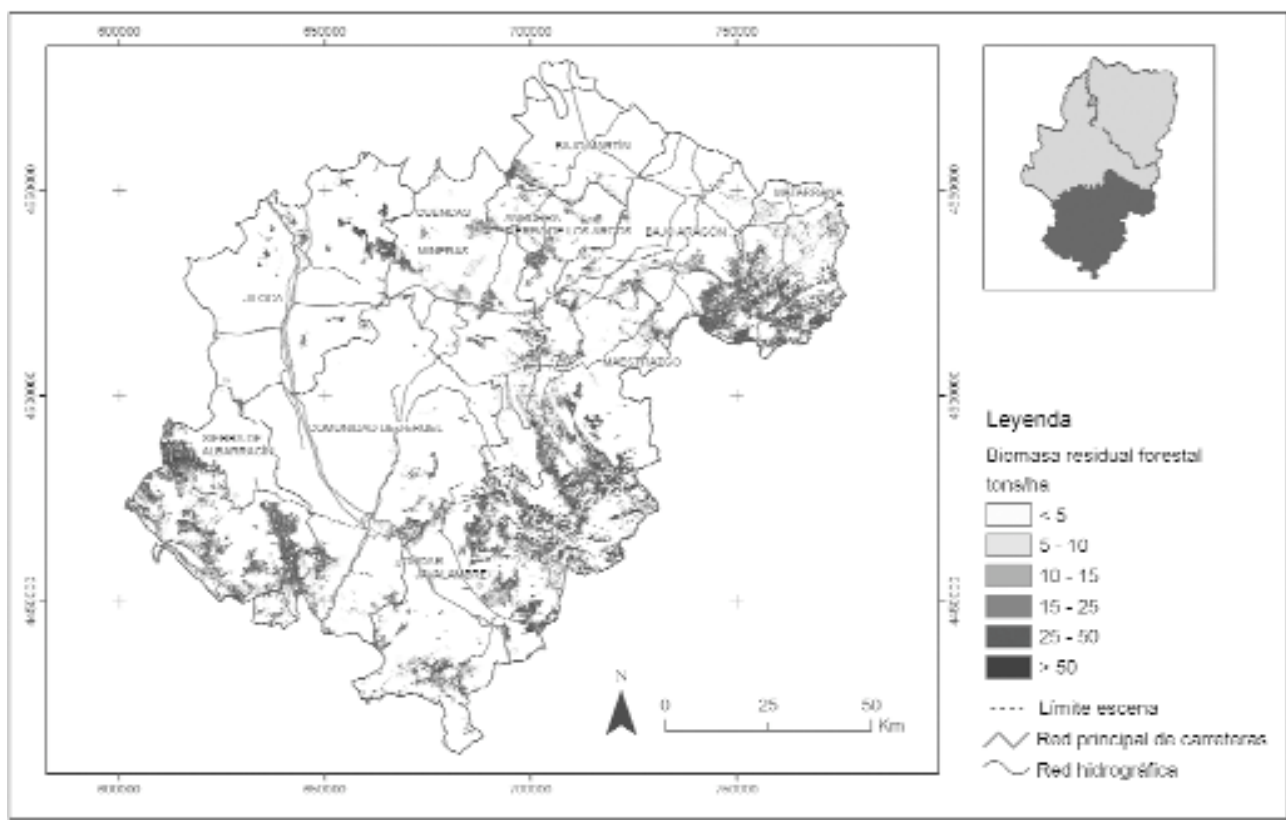

Figura 1. BRF en la provincia de Teruel (tons/ha) (julio 2008).

\section{Conclusiones}

Este trabajo ha mostrado la utilidad de la teledetección para inventariar la cantidad de residuos energéticamente aprovechables que se pueden obtener de los pinares turolenses. Para ello, las tres fuentes de información típicamente usadas para estimar biomasa (datos de muestreo en el campo, imágenes de satélite e información auxiliar; $\mathrm{Lu}, 2006$ ) han sido tratadas de forma cuidadosa teniendo en cuenta las características específicas de los bosques mediterráneos. Así, para reducir el problema que la heterogeneidad de éstos introduce en el establecimiento de modelos estimativos, se han aplicado tres métodos distintos de extracción de la información espectral, 
resultando el que combina la utilización de ventanas de 3x3 píxeles y el CV el más adecuado, al ser capaz de aislar un grupo homogéneo de parcelas para ajustar ecuaciones de regresión con un elevado $\mathrm{R}^{2}$ y representativo de todo el área de estudio. Las razones por las que el método de análisis visual no ha logrado tan buenos resultados pueden estar relacionadas con imprecisiones en la delimitación de las nuevas áreas homogéneas y con que en éstas se asume un valor constante de BRF para toda el área a partir de una localización puntual. Por su parte, la menor adecuación del método de segmentación espectral puede relacionarse con que ninguna de las abordadas ha modelado adecuadamente el patrón espacial de distribución de la BRF.

Finalmente, señalar que para mejorar los resultados es necesario investigar el uso de nuevos métodos que permitan modelizar de forma más adecuada la heterogeneidad para conseguir modelos más explicativos con un mayor número de parcelas. El uso de algoritmos de segmentación implementados en programas como eCognition puede permitir delimitar regiones espectrales más relacionadas con el patrón forestal que las que aquí se han utilizado.

\section{Bibliografía}

Alonso, E., Asín, J. y Pascual, J. (2005). Biomasa residual forestal: regresiones para las especies del género Pinus existentes en la provincia de Teruel. En SECF (eds) La ciencia forestal: respuestas para la sostenibilidad, Zaragoza, SECF (CD.Rom).

Colby, J.D. (1991). Topographic normalization in rugged terrain. Photogrammetric Engineering and Remote Sensing, 57, 531537.

Esteban, L.S., Pérez, P., Ciria, P. y Carrasco, J.E. (2004). Evaluación de los recursos de biomasa forestal en la provincia de Soria. Madrid, CIEMAT.

Franklin, S.E. (2001). Remote sensing for sustainable forest management. New York, Taylor \& Francis-CRC Press.

García Martín, A. (2009). Estimación de biomasa residual mediante imágenes de satélite y trabajo de campo. Modelización del potencial energético de los bosques turolenses. Tesis doctoral, Universidad de
Zaragoza (http://zaguan.unizar.es/record/ 4570 ? $\ln =\mathrm{es})$.

García-Martín, A., de la Riva Fernández, J., Pérez-Cabello, F., Montorio-Llovería, R., García Galindo, D. y Pascual Puigdevall, J. (2008). Evaluation of the effect of temporality on forest residual biomass estimation using summer Landsat TM imagery. En Proceedings of the 16th European Biomass Conference and Exbibition, Valencia, ETARenewable Energies, pp. 254-262.

Hair, J.F., Anderson, R.E., Tatham, R.L. y Black, W.C. (1999). Análisis multivariante. Madrid, Prentice Hall Iberia.

IDAE. (2005). Plan de Energías Renovables en España 2005-2010. Madrid, Instituto para la Diversificación y Ahorro de la Energía, Ministerio de Industria, Turismo y Comercio.

Katila, M. y Tomppo, E. (2001). Selecting estimation parameters for the Finnish multi- 
source national forest inventory. Remote Sensing of Environment, 76, 16-32.

Lu, D. (2006). The potential and challenge of remote sensing-based biomass estimation. International Journal of Remote Sensing, 27, 1297-1328.

Mäkelä, H. y Pekkarinen, A. (2001) Estimation of timber volume at the sample plot level by means of image segmentation and Landsat TM imagery. Remote Sensing of Environment, 77, 66-75.

Mäkelä, H. y Pekkarinen, A. (2004) Estimation of forest stand volumes by Landsat TM imagery and stand-level fieldinventory data. Forest Ecology and Management, 196, 245-255.

McRoberts, R.E. y Tomppo, E.O. (2007) Remote sensing support for national forest inventories. Remote Sensing of Environment, 110, 412-419.

Muukkonen, P. y Heiskanen, J. (2005). Estimating biomass for boreal forests using ASTER satellite data combined with standwise forest inventory data. Remote Sensing of Environment, 99, 434-447.

Pascual, J., García, D. y García-Martín, A (2007). Optimum stands for forest residual biomass harvesting: development of a spatial index. Proceedings of the 15th European Biomass Conference and Exhibition, Florence, ETA, p. 353-360.

Richardson, J., Björheden, R., Hakkila, P., Lowe, A.T. y Smith C.T. (2002). Bioenergy from Sustainable Forestry: Guiding Principles and Practice. Dordrecht, Kluwer Academic Publishers.

Salvador, R. y Pons, X. (1998). On the applicability of Landsat TM images to Mediterranean forest inventories. Forest Ecology and Management, 104, 193-208.

Shoshany, M. (2000). Satellite remote sensing of natural Mediterranean vegetation: a review within an ecological context. Progress in Physical Geography, 24, 153158.

Tokola, T., Pitkänen, J., Partinen, S. y Muinonen, E. (1996). Point accuracy of a nonparametric method in estimation of forest characteristics with different satellite materials. International Journal of Remote Sensing, 17, 2333-2351.

Velázquez, B. (2006). Situación de los sistemas de aprovechamiento de los residuos forestales para su utilización energética. Ecosistemas, 15, 77-86. 